\title{
5. COMMISSION DES ANALYSES DE TRAVAUX ET DE BIBLIOGRAPHIE
}

Président: M. P. Bourgeois.

Membres: MM. Baldet, Bouska, Brasch, Collinder, Dermul, Gastardi, Guyot, Heinemann, Kopff, Kulikovsky, Lundmark, Martynov, Neubauer $\uparrow$, Nordenmark, Pogo, Sconzo, Selga, Witkowski, Zagar.

Dans le cadre des questions étudiées et des résolutions adoptées par la Commission lors de l'Assemblée générale de Rome en I952:

I. L'Astronomisches Rechen-Institut a édité les volumes 49 (année r949), 50 (année I950), 5I (année I95I) du Jahresbericht. Les dispositions sont prises pour que les volumes 52 à 55 (années 1952-55) paraissent respectivement en mai et décembre I955, en juillet 1956 et en avril I957. Grâce à une intervention financière de l'U.N.E.S.C.O. en I953, tous les volumes importants du Jahresbericht y compris le volume 5I peuvent être mis temporairement à la disposition des intéressés à prix réduit.

2. La publication des Extraits d'Astronomie du Bulletin Analytique du C.N.R.S. qui font suite à la Bibliographie mensuelle de l'astronomie, s'est poursuivie régulièrement. Le service en a été fait gratuitement à I4I bibliothèques d'observatoires et d'instituts intéressés. Une table des auteurs et des matières a été publiée pour l'année I953.

3. L'Institut d'Information scientifique de l'Académie des Sciences d'U.R.S.S. a entrepris, à partir d'octobre I953, la publication d'une revue mensuelle de bibliographie sous le titre: Referativny Journal-Astronomie et Géodésie. Les analyses qui y sont données sont détaillées et concernent les travaux publiés dans tous les pays. Un index récapitulatif sera publié chaque année.

4. La publication des Astronomical Neros Letters (A.N.L.) diffusant des résumés de travaux astronomiques publiés uniquement en langue russe a été poursuivie. Le Prof. Struve ayant dû renoncer, en juin I953, à l'absorbante tâche d'éditeur en chef, le Prof. Kourganoff a repris sa succession au Ier janvier 1954. Le Centre National Français de la Recherche Scientifique (C.N.R.S.) assure au Prof. Kourganoff un crédit spécial destiné à couvrir une partie des frais de secrétariat. I52 exemplaires des A.N.L. sont distribués gratuitement aux bibliothèques des Observatoires. Les numéros 74 et 75 sont à la reproduction. Un numéro spécial (73 bis) a été consacré à l'ensemble des travaux relatifs à la formation d'étoiles à partir de filaments des nébuleuses diffuses.

5. Le travail entrepris pour combler la lacune entre la Bibliographie Houzeau-Lancaster et le Jahresbericht a été continué régulièrement à l'intervention du Comité National Belge d'Astronomie. Le travail touche à sa fin.

6. La réédition du répertoire Les observatoires astronomiques et les astronomes dont M. Rigaux est chargé par l'Observatoire royal de Belgique pourra être envisagée prochainement, la rédaction du manuscrit étant en voie d'achèvement.

\section{P. BOURGEOIS \\ Président de la Commission}

\section{Procès-verbal des séances $d u$ 3I août et $d u$ I septembre}

PrÉsident: M. P. Bourgeois.

SeCrétaire: M. A. Dermul.

\section{Séance $d u$ 3I août}

Sont présents, outre le président et le secrétaire:

MM. F. Baldet, D. Belorizky, B. Bok, W. Fricke, V. Kournagoff, P. G. Kulikovsky, W. Lohmann, J. Witkowski, Fr. Zagar.

Le président attire l'attention des membres présents sur l'excellent Reference Journal publié trimestriellement en Union Soviétique depuis octobre I953 et consacré à la 
bibliographie de la littérature astronomique courainte. Quelques fascicules déposés sur le bureau permettent aux membres de se rendre compte de l'importance de cette publication.

Il annonce que la subvention de 600 dollars, accordée par l'U.A.I. lors de son Assemblée de Rome (I952) pour la traduction des plus importants travaux astronomiques en langue russe ainsi que pour la publication des $A$ stronomical $N$ ews Letters, a été affectée intégralement à cette dernière: il rend hommage au travail appréciable fourni dans ce domaine par le Prof. V. Kourganoff.

Le Dr W. Fricke fait savoir que le comité d'édition de l'Astronomischer Jahresbericht a décidé de faire paraître dorénavant ses volumes annuels vers la fin de l'année suivant celle de la date des publications répertoriées. Il insiste pour que l'U.A.I. recommande à nouveau à tous les observatoires et à tous les astronomes d'envoyer directement un exemplaire de leurs publications à la direction du Jahresbericht aussitôt qu'elles seront sorties de presse, et non plus par la voie, plus lente, des organismes généraux de distribution. La Commission discute des meilleurs moyens à mettre en œuvre pour obtenir que cette recommandation devienne efficace et, après avoir décidé de demander au secrétaire général de l'Union d'envoyer une circulaire à ce sujet à tous les membres, elle adopte la résolution no. $I$.

Le Président distribue des exemplaires du rapport U.N.E.S.C.O. de Mlle Paule Salvan sur Les conditions de l'organisation bibliographique de deux domaines particuliers: astronomie et urbanisme. Il prie les membres de vouloir bien examiner ce travail en vue de la discussion à laquelle celui-ci devra donner lieu à la séance du jeudi Ier septembre.

Il en est fait de même pour l'édition provisoire (miméographiée) du nouveau répertoire: Les observatoires astronomiques et les astronomes.

On aborde ensuite le sujet du subside à accorder à la commission d'édition des Astronomical Neres Letters. Cette publication commencée par le Prof. O. Struve et abandonnée par celui-ci en I953 en raison de ses occupations professionnelles, est assurée depuis lors par le Prof. Kourganoff, directeur de l'Institut d'astronomie de l'Université de Lille.

Pour en assurer la continuation, dit le Prof. Kourganoff, il est indispensable de pouvoir disposer d'un crédit annuel pour couvrir les frais de secrétariat et de distribution, frais qu'il estime se monter à un minimum de 1500 dollars par an. Il fait un long exposé des difficultés rencontrées dans leur publication, difficultés principalement d'ordre financier: les frais entraînés par la publication et la distribution peuvent être évalués à 7 ou 8 dollars par an et par observatoire. En plein accord avec le Prof. B. Bok, avec lequel il a d'ailleurs longuement examiné cette question, il demande que soit mise à sa disposition une subvention de 4500 dollars pour la période des trois années à venir.

Le Prof. Bok, après avoir fait l'historique des résumés des travaux russes, appuie entièrement la proposition du Prof. Kourganoff et tient à remercier plus spécialement Mlle Margaret Olmsted, de l'Observatoire de Harvard College, pour le travail consciencieux qu'elle a fait pendant plusieurs années pour cette œuvre si utile.

On discute le point de savoir s'il est utile ou désirable de faire payer une légère rétribution par les institutions qui reçoivent les Astronomical News Letters, ceci en vue de réduire les charges financières. Les arguments mis en avant étant généralement défavorables à cette mesure, il sera continué à faire le service gratuit de cette publication à deux cents observatoires.

Le Président rend hommage à MM. Struve, Bok et Kourganoff, ainsi qu'à Mlle Olmsted pour tout le travail utile qu'ils ont fourni. La Commission se met d'accord pour demander à l'U.A.I. une subvention annuelle de $\mathrm{I}_{500}$ dollars pour les trois années à venir et vote à l'unanimité la résolution no. 2 . 


\section{Séance $d u$ I septembre}

Sont présents, outre le président et le secrétaire:

MM. F. Baldet, A. Beer, Ch. Bertaud, B. Bok, W. Fricke, P. Kulikovsky, W. Lohmann, C. Luplau Jansen, Mlle R. Northcott, MM. P. Sconzo et J. Witkowski.

Le secrétaire, chargé par le Comité National Belge sous les auspices de l'U.A.I. de la coordination et de la publication de la Bibliographie de l'astronomie pour la période I88I-98, fait rapport sur l'état d'avancement des travaux. Depuis la dernière assemblée de l'Union (Rome, I952), le contact a été établi ou a été renoué là où il avait été interrompu, avec quinze pays. Deux de ceux-ci ont fait savoir qu'ils n'ont pas publié de travaux astronomiques pendant le période sous considération. Parmi les treize autres, onze ont fait parvenir leur contribution, soit totale, soit partielle, constituant un ensemble de 4956 fiches, ce qui porte à près de 32,00o le nombre de fiches réunies à ce jour. La Commission est heureuse d'apprendre que parmi ces rentrées figure la contribution importante de l'U.R.S.S., apportée au Congrès par les délégués russes et que le Prof. A. Kahrstedt a fait savoir que, de retour dans son pays, il fera parvenir un premier envoi de deux à trois cents fiches du travail entrepris en Allemagne.

Quant aux dix-sept pays avec lesquels il n'a pas encore été possible d'établir le contact, plusieurs membres font d'utiles suggestions: ils sont d'ailleurs d'avis que plusieurs de ces pays n'auront pas eu de littérature astronomique en 188I-98. D'autre part, le Prof. P. Sconzo, chef du département des calculs à l'Observatoire de La Plata, s'offre spontanément pour faire le dépouillement des publications de toute l'Amérique du Sud et Mlle Ruth J. Northcott se met gracieusement à la disposition de la Commission pour faire le même travail en ce qui concerne son pays, le Canada.

Grâce à ces aides précieuses, on peut entrevoir avec confiance la fin prochaine du dépouillement de l'ensemble de la littérature astronomique de la période I88I-98 et le président propose d'envoyer au Comité exécutif la résolution no. 3, disant que la Commission insiste pour que les dispositions nécessaires soient prises pour terminer le manuscrit avant la prochaine assemblée de l'U.A.I. Cette résolution est adoptée à l'unanimité.

Revenant sur la question de la publication du Jahresbericht, le Dr Fricke propose que le président se mette en rapport avec les éditeurs des grandes bibliographies astronomiques actuelles afin d'obtenir qu'ils échangent mutuellement leurs publications. Le président fait remarquer que le Bureau des comptes-rendus analytiques du Centre international des unions scientifiques (Abstracting Board de l'I.C.S.U.) essaie précisément de coordonner les grandes bibliographies scientifiques et qu'il est donc possible de voir se réaliser quelque chose d'utile dans cette voie.

Le Prof. Fricke exprime sa gratitude envers le Président de la Commission 5 à propos de la subvention accordée par l'U.N.E.S.C.O. pour la publication du Jahresbericht. Le prix de souscription à cette bibliographie, qui avait été arrêté à $50 \mathrm{DM}$. par volume, a pu être réduit à $30 \mathrm{DM}$. pour les membres de l'Union, jusque, et y compris, le vol. 52 . A partir du vol. 53, l'U.N.E.S.C.O. ayant cessé sa subvention, on espère toutefois pouvoir fixer le prix à 40 D.M

M. Baldet fait savoir que la publication du Bulletin analytique $d u$ C.N.R.S., dont les Extraits d'astronomie mensuels font suit à la Bibliographie mensuelle de l'astronomie avec laquelle ils ont fusionné en r948, s'est poursuivie régulièrement depuis la dernière assemblée de l'U.A.I. à Rome en I952. Conformément à l'adoption par cette assemblée générale de la résolution no. 5 de la Commission 5 (subvention de 1500 fr.-or à la B.M.A. pour les années I952, I953 et I954), le C.N.R.S. a assuré la publication et le service gratuit des Extraits d'astronomie pendant toute l'année I953 à I4I bibliothèques d'observatoires et de grands établissements scientifiques intéressés. Il a obtenu ainsi un supplément de trente-deux abonnements, ce qui porte maintenant à $\mathrm{I}_{44}$ le nombre total des abonnés.

Ce nombre est encore insuffisant et l'effort doit être poursuivi au cours de la prochaine période triennale. En conséquence il y a lieu de demander à l'U.A.I. le renouvellement de la subvention de 1500 fr.-or pour les trois années I955, I956 et I957, ce qui permettrait d'assurer à nouveau un service gratuit d'un an des Extraits d'astronomie aux établissements scientifiques intéressés. 
Il y aurait lieu de rappeler que l'envoi au C.N.R.S., Bulletin analytique, 45, rue d'Ulm, Paris-Ve, d'un exemplaire des publications astronomiques dès leur parution, est hautement désiré.

La Commission décide à l'unanimité d'envoyer à ce sujet au Comité exécutif la résolution no. 4 .

Le Président précise qu'à défaut de crédit l'Observatoire royal de Belgique n'a pas encore pu mettre à l'impression le Répertoire: Les observatoires astronomiques et les astronomes, mais qu'il présente aux membres de la Commission une édition provisoire, miméographiée, sur laquelle il demande leur avis. L'année prochaine, l'Observatoire disposera des crédits nécessaires et pourra donc faire imprimer le volume; des dispositions sont prises pour que toutes les institutions et les personnes qui y sont mentionnées aient encore l'occasion de compléter ou éventuellement de rectifier la notice qui leur y est consacrée.

L'U.A.I. a accordé pour ce travail une subvention de 500 dollars, dont une faible partie seulement a été utilisée, mais qui reste à la disposition de l'Observatoire royal de Belgique. Il demande à l'assemblée si elle est d'accord pour demander une seconde et dernière subvention de 500 dollars pour l'achèvement du travail. La réunion décide à l'unanimité d'introduire à ce. sujet la résolution no. 5. Le volume sera envoyé gratuitement aux observatoires et institutions scientifiques.

M. le Prof. F. E. Brasch a dressé le catalogue des ouvrages de Newton existant à la bibliothèque de la Stanford University. Ce catalogue compte environ 3000 numéros et M. Brasch espère pouvoir le publier aussitôt qu'il disposera des fonds nécessaires. Le Président propose de féliciter M. Brasch de l'initiative qu'il a eue d'entreprendre cet utile travail de bibliographie scientifique.

Quant au rapport de Mlle P. Salvan, distribué lors de la séance précédente, la Commission en prend acte, mais comme les membres ont disposé de trop peu de temps pour en prendre connaissance, aucune discussion ne s'engage: le Prof. Fr. Zagar fait observer qu'on n'a pas demandé l'opinion de l'Italie sur cette publication.

Pour ce qui est des bibliographies spéciales, se rapportant à des sujets particuliers, et au sujet desquelles le Prof. D. Martynov avait fait une proposition lors de l'Assemblée de l'U.A.I. à Rome, en I952, le président, arrivé au terme de son mandat, exprime l'espoir que ce travail pourra être mené à bonne fin par son successeur. Il remercie tous ceux qui l'ont efficacement aidé pendant toute la durée de son mandat et souhaite la bienvenue aux membres cooptés qui viendront s'ajouter à la Commission 5 .

\section{Résolutions}

I. La Commission 5 prie instamment les institutions et les personnes publiant un travail concernant l'astronomie de bien vouloir en faire parvenir au moins un exemplaire, aussi rapidement que possible et sans passer par les lents services généraux de distribution, aux adresses ci-après, des bibliographies astronomiques.

(a) The Editor of the Astronomischer Jahresbericht, Astronomisches Rechen-Institut, I4 Grabengasse, Heidelberg, Germany.

(b) The Editor of the Referativny Žurnal, Astronomija i Geodesia, Moskva D-219, Baltijskij Pos. D. $42 \mathrm{~B}$.

(c) L'éditeur du Bulletin Analytique du C.N.R.S., 45 rue d'Ulm, Paris-Ve, France.

(d) L'éditeur des Astronomical News Letters, Laboratoire d'astronomie, Porte de Douai, Lille (Nord), France.

2. La Commission 5 prie l'U.A.I. de prévoir pour la prochaine période de trois ans, annuellement, une somme de 1500 dollars (crédit total 4500 dollars) de manière à assurer la continuation de la publication des Astronomical News Letters sous la direction du Dr V. Kourganoff du Laboratoire d'astronomie de l'Université de Lille. Il est entendu que le Dr Kourganoff, d'accord avec le Président de la Commission 5, préparera une liste des instituts astronomiques qui recevront gratuitement les $A . N . L$. Le nombre total des instituts 
qui recevront de cette manière les $A . N . L$. ne dépassera pas, dans le cadre du crédit prévu ci-dessus, deux cents.

3. La Commission témoigne sa satisfaction quant à l'état d'avancement du travail entrepris pour combler la lacune entre la Bibliographie générale de l'Astronomie de Houzeau et Lancaster et l'Astronomischer Jahresbericht, et insiste pour que les dispositions nécessaires soient prises pour terminer le manuscrit avant la prochaine Assemblée générale de l'U.A.I.

4. La Commission, appréciant l'étendue de la diffusion donnée aux Extraits d'astronomie $d u$ Bulletin analytique du C.N.R.S., estime que la subvention qui a été donnée à celui-ci doit être renouvelée à concurrence de 1500 francs-or pour la période des trois années à venir (1955-56-57).

5. La Commission ayant pris connaissance de l'édition provisoire du Répertoire: Les observatoires astronomiques et les astronomes, est heureuse des résultats atteints et estime qu'il $\mathrm{y}$ aura lieu d'attribuer à l'Observatoire royal de Belgique une seconde et dernière subvention de 500 dollars pour permettre d'entreprendre l'édition définitive de cet ouvrage. 